\title{
LA EMPRESA SOCIAL: ACTOR CLAVE EN LA ECONOMÍA SOCIAL Y SOLIDARIA
}

\section{THE SOCIAL ENTERPRISE: KEY ACTOR IN THE SOCIAL AND SOLIDARY ECONOMY}

\author{
Karina Eréndira Torres González*
}

*Doctorando en Ciencias en Negocios Internacionales en Instituto de Investigaciones Económicas y Empresariales en ININEE- UMSNH. ORCID: https://orcid.org/0000-0003-1280-5941.

Dirección para correspondencia: LM.Erendira.TG@gmail.com 


\section{RESUMEN}

OBJETIVO: Analizar los aportes de la empresa social a la economía social y solidaria; se eligió como objeto de estudio una asociación de empresas sociales fundada en un pequeño municipio del estado de Michoacán que promueve el desarrollo de este tipo de economía.

MATERIAL Y MÉTODO: Se empleó el método cualitativo, en específico el estudio de casos, el cual es una descripción y análisis a detalle de unidades sociales (Yin, 1992). El material de investigación fue la observación en campo y entrevistas a profundidad, no estructuradas, semiestructuradas y estructuradas.

RESULTADOS: Existen ciertas divergencias entre los objetivos que la economía social marca para las empresas sociales y las empresas que pertenecen a la asociación que fue objeto de estudio. No obstante, se encontraron otros casos en los que abonan al proceso de desarrollo local.

CONCLUSIONES: La empresa social favorece que productores y consumidores establezcan relaciones de reciprocidad en pro de sus comunidades y el medio ambiente. Se puede concluir que la economía social y solidaria encuentra en este tipo de empresas un vínculo primordial entre productor y consumidor que permite promover la práctica y desarrollo de este tipo de economía.

PALABRAS CLAVE: Economía social y solidaria. Empresa social. Desarrollo local.

\section{ABSTRACT}

OBJECTIVE: To analyze contributions of the social enterprise in the social and solidary economy; the object of study was an association of social enterprises founded in a small municipality in Michoacan that promotes the development of this type of economy.

MATERIAL AND METHOD: A qualitative approach with a case study technique was performed, which is a detailed description and analysis of social unities (Yin, 1992). The material of research was observation in field and in-depth interviews, non-structured, semi-structured and structured. 
LA EMPRESA SOCIAL: ACTOR CLAVE EN LA ECONOMÍA SOCIAL Y SOLIDARIA.

RESULTS: There are certain divergences between the objectives the social economy indicates for social enterprises and the companies belonging to the association were subjected to study. Nevertheless, other cases were found and contribute to the local development.

CONCLUSIONS: The social enterprise favors that producers and consumers establish reciprocity relations in benefit of their communities and the environment. It can be concluded that the social and solidary economy find a bond in this type of companies primarily between the producer and the consumer that allows the fostering of practice and development of this type of economy.

KEY WORDS: Social and solidary economy. Social enterprise. Local development.

\section{INTRODUCCIÓN}

Las producción, consumo y distribución son actividades propias de la economía, mismas que de acuerdo con diversos autores se han dirigido a una concentración y acumulación de capital en el sistema económico actual. Existen diferentes definiciones sobre el concepto de economía. No obstante, en conjunto dichas definiciones podrían resumirse de la siguiente manera, según Collin (2014, p. 9); "la economía es el arte de satisfacer las necesidades de la gente". En ese sentido, la discusión sobre el actuar de la economía actual emerge al cuestionar ¿En qué momento la economía dejó de preocuparse por satisfacer las necesidades de la gente y optó por únicamente acumular y generar dinero o bien riqueza? Esta situación ha perjudicado o dejado al margen a cierta parte de la población, con la característica principal de ser la más vulnerable. En ese contexto, surge la economía social y solidaria, como una alternativa que apuesta por una nueva forma de hacer economía, en otras palabras; una forma diferente de producir, consumir y distribuir (Razeto, 2014). Uno de los objetivos de la economía social y solidaria es crear empresas que se diferencien de las capitalistas que solo buscan "maximizar el beneficio" y la "acumulación de capital" usando a las personas como objetos para conseguirlo, sin olvidar el daño ecológico que causan con su actividad. Estas empresas alternativas son llamadas empresas sociales, quienes tienen por principal objetivo producir productos y servicios que sean de utilidad colectiva, y que respeten el medio ambiente del cual todos los seres humanos dependemos. Dichas empresas tienen características específicas que las diferencian del resto. 
LA EMPRESA SOCIAL: ACTOR CLAVE EN LA ECONOMÍA SOCIAL Y SOLIDARIA.

\section{Economía Social y Solidaria, ¿Otra economía?}

Existen economías diferentes al capitalismo, entre las cuales se encuentran la economía social y solidaria, la economía para la vida y la economía ecológica, por ejemplo. Este tipo de economías comparten la idea de un mundo que supere la visión capitalista y coloque a la reproducción de la vida y la solidaridad humana como el objetivo primordial de la economía; la economía social existe no sólo como propuesta sino como experiencias en América Latina y el mundo, bajo la convicción de que "otra economía es posible" como lo define el Foro Social Mundial (Gonzáles, 2011). Se apuesta por una economía alternativa al sistema capitalista, que pretende un cambio estructural en el cual se prioriza la vida de las personas y el cuidado del planeta.

La economía solidaria nace del tronco común de la economía social. De acuerdo con Tapia (2012), este tipo de economía es una manera distinta de hacer economía, más justa y humana, de generar recursos con base en valores, tales como; la solidaridad, la reciprocidad y la cooperación, respetando el medio ambiente y persiguiendo el beneficio colectivo. La Economía Solidaria tiene por objetivo construir relaciones de producción, distribución, consumo y financiación basadas en la justicia, la cooperación, la reciprocidad, y la ayuda mutua. Frente al capital y su acumulación, la economía solidaria pone a las personas y su trabajo en el centro del sistema económico, y otorga a los mercados un papel instrumental siempre al servicio del bienestar de todas las personas y de la reproducción de la vida en el planeta (Pérez de Mendiguren, Etxarri \& Guridi Aldanondo, 2008). En ese sentido, para José Luis Coraggio (2016) la economía social es un concepto que pretende superar la opción entre el mercado capitalista y un Estado central planificador y regulador de la economía. Plantea que el mercado capitalista debe ser superado con límites sociales y si es posible, generar mercados donde los precios y las relaciones son resultado de una matriz social que pretende la integración con esfuerzo y resultados distribuidos de manera más igualitaria. Laura Collin (2008), considera a la economía social como una economía asociativa donde sus participantes, es decir productores y consumidores, se asocian con el fin de satisfacer sus necesidades, y propone una relación diferente a la de la producción por contrato, orientada por el afán de maximizar la ganancia. Por otra parte, al concepto de economía social se le agrega el adjetivo "solidaria" puesto que hace referencia a actividades diseñadas para experimentar nuevos modelos de funcionamiento de la economía, tales como el Comercio Justo (García, 2011). En ese sentido, según Collin (2008) existen al menos tres tipos de solidaridades: la solidaridad con los seres humanos, con la naturaleza y con la cultura. La solidaridad humana se refiere a la reproducción de las bases para 
LA EMPRESA SOCIAL: ACTOR CLAVE EN LA ECONOMÍA SOCIAL Y SOLIDARIA.

crear nuevos empleos, para satisfacer la necesidad de trabajo y en solidaridad con las generaciones futuras; la solidaridad con la naturaleza se refiere a aprovechar los recursos naturales, preocuparse por su reproducción y por tanto su conservación. La solidaridad con la cultura, expone la importancia del patrimonio cultural, de las creaciones humanas y fundamentalmente de la diversidad de gustos, dentro de la economía social ésta debería respetarse y plasmarse en las necesidades y posibilidades de producción locales. En la economía solidaria el proceso de acumulación tiene una característica importante, la acumulación es solidaria, por lo tanto, justa y equitativa. Desde ésta perspectiva según las autoras Collin y Torres (2007) las empresas deberían presentar ciertas características que las distingan como empresas sociales. The Social Business Initiative (SBI) (2018) perteneciente a la Comisión Europea define a la empresa social como "un instrumento de la economía social cuyo objetivo principal es generar impacto social a diferencia de sólo obtener ganancias para sus propietarios o accionistas". Este tipo de empresa ofrece bienes y servicios para el mercado de forma emprendedora e innovadora y emplea las ganancias fundamentalmente para lograr objetivos sociales; su gestión es transparente y responsable, además tiene la característica de involucrar a empleados, consumidores y otros actores en sus actividades comerciales. Las empresas sociales se distinguen del resto debido a que presentan características particulares que las distinguen como empresas sociales (ver tabla 1):

\section{Tabla 1}

Características de las empresas sociales

\begin{tabular}{|c|c|}
\hline Característica & Elementos \\
\hline $\begin{array}{l}\text { 1. Cuidado del medio } \\
\text { ambiente. }\end{array}$ & $\begin{array}{l}\text { - Protección del medio ambiente. } \\
\text { - Reposición de insumos renovables. } \\
\text { - Reciclamiento de residuos fuentes energéticas alternativas. } \\
\text { - Protección al consumidor. }\end{array}$ \\
\hline $\begin{array}{l}\text { 2. Desarrollo local de zonas } \\
\text { desfavorecidas. }\end{array}$ & $\begin{array}{l}\text { - Empresas sociales en zonas rurales remotas. } \\
\text { - Desarrollo de barrios. } \\
\text { - Planes de rehabilitación en zonas urbanas. } \\
\text { - Ayuda al desarrollo y cooperación al desarrollo con terceros países. }\end{array}$ \\
\hline 3. Democracia interna. & $\begin{array}{l}\text { - Una persona un voto. } \\
\text { - Democracia directa en cada nivel. } \\
\text { - Transparencia dentro del grupo, comunidad. }\end{array}$ \\
\hline $\begin{array}{l}\text { 4. Servicios sociales } \\
\text { personales. }\end{array}$ & $\begin{array}{l}\text { - Salud, bienestar y atención médica. } \\
\text { - Capacitación profesional, educación, servicios de salud. } \\
\text { - Servicios de cuidado infantil. } \\
\text { - Servicios para personas mayores o ayuda para personas desfavorecidas. }\end{array}$ \\
\hline 5. Integración laboral. & - Formación e integración de personas con discapacidad y desempleadas. \\
\hline
\end{tabular}

(continúa) 
LA EMPRESA SOCIAL: ACTOR CLAVE EN LA ECONOMÍA SOCIAL Y SOLIDARIA.

Tabla 1

Características de las empresas sociales

\begin{tabular}{|c|c|}
\hline Característica & Elementos \\
\hline 6. Remuneración equitativa. & $\begin{array}{l}\text { - En relación al esfuerzo y capacidades } \\
\text { - Equidad de género. }\end{array}$ \\
\hline 7. Constitución legal. & $\begin{array}{l}\text { - No existe una forma legal única para las empresas sociales. } \\
\text { - Empresas sociales operan en forma de cooperativas sociales. } \\
\text { - } \text { - Otgunas están registradas como empresas privadas limitadas por garantía. } \\
\text { como sociedades de previsión, asociaciones, organizaciones voluntarias, } \\
\text { organizaciones benéficas o fundaciones. }\end{array}$ \\
\hline $\begin{array}{l}\text { 8. Formas alternativas de } \\
\text { circulación. }\end{array}$ & $\begin{array}{l}\text { - Compra de insumos en moneda alternativa } \\
\text { - Pago del trabajo y por tanto promoción del consumo en moneda alternativa. }\end{array}$ \\
\hline $\begin{array}{l}\text { 9. Valor cultural incorporado al } \\
\text { producto. }\end{array}$ & $\begin{array}{l}\text { - Técnicas tradicionales. } \\
\text { - Diseños y materiales. } \\
\text { - Valor simbólico. } \\
\text { - Valor de uso. }\end{array}$ \\
\hline
\end{tabular}

Fuente: Elaboración propia con base en Collin y Torres (2007), European Commission, (2011), Vega (2014) y Gonzáles Butrón (2011).

Las empresas sociales presentan principalmente las características resumidas en la tabla 1, puesto que son empresas que buscan diferenciarse del resto. Aunque las opiniones pueden variar (Díaz Foncea \& Marcuello, 2014), (Girona \& Sanchis Palacio, 2014) y (Monzón, 2006), al final coinciden en que las empresas sociales y solidarias se dirigen a la producción de bienes y servicios de utilidad colectiva, que buscan satisfacer las necesidades e intereses comunes que las empresas privadas y/o el Estado no logran o lo hacen de manera insuficiente. Estas organizaciones se centran en objetivos sociales, ambientales o comunitarios más amplios. Lo anterior es de gran relevancia debido a que se pretende no sólo producir cualquier cosa con tal de obtener dinero (Collin y Torres, 2007), sino producir de manera responsable bienes y servicios útiles y beneficiosos. La economía social y solidaria puede ser confundida con ciertas prácticas que realizan las empresas tradicionales con el fin de resarcir de alguna forma los efectos negativos de su actividad en el entorno social, ambiental y económico. Estas actividades son; la Responsabilidad Social Empresarial (RSE), el emprendimiento tradicional y las políticas públicas convertidas en programas sociales que tienen por objetivo aminorar los efectos de la pobreza. Sin embargo, estas actividades tienen marcadas diferencias que las distinguen de las empresas sociales sobre todo en lo que respecta a los valores, por lo cual no pueden ser consideradas economía social. En ese sentido, la economía social y solidaria a diferencia de otro tipo de economías tiene fundamentos, principios y valores que la caracterizan; estos mismos valores son la base sobre la cual se promueve su práctica como son; la igualdad, el empleo, el medio TORRES-GONZÁLEZ K. E. 
LA EMPRESA SOCIAL: ACTOR CLAVE EN LA ECONOMÍA SOCIAL Y SOLIDARIA.

ambiente, la cooperación, el compromiso con el entorno, y por último la autogestión y toma de decisiones participativa. Estos valores resultan un eje toral sobre el cual actúan las empresas sociales para diferenciarse del resto y son la base sobre la cual este tipo de empresas realizan sus actividades comerciales y de producción, a diferencia de las empresas capitalistas las empresas sociales operan bajo principios y valores.

\section{MATERIAL Y MÉTODO}

En esta investigación se empleó el estudio de casos, el cual es un "método de investigación de tipo cualitativo, que se utiliza para obtener una profunda comprensión de la realidad social" (Barrio del Castillo et al., 2016). Yin (1992) indica que "el estudio de caso es una descripción y análisis a detalle de unidades sociales o entidades educativas", el estudio de caso no tiene especificidad, por lo que puede emplearse en cualquier disciplina para dar respuesta a las preguntas de investigación. Para obtener la información, se realizó previamente observación en campo durante reuniones organizadas por el gobierno del estado de Michoacán para promover emprendimientos locales, derivado de información recabada al asistir a dichas ferias se identificaron informantes clave que participan en ferias o encuentros que promueven la economía social y solidaria. Una vez identificado el objeto de estudio, se procedió a elaborar un guion de entrevista estructurada y semiestructurada a fin de obtener la mayor información posible y cuidando no sesgar la investigación cualitativa. Con base en la información obtenida de entrevistas no estructuradas realizadas durante el primer acercamiento y observación en campo, se detectaron 3 principales organizaciones que promueven la economía social, no obstante, se eligió para el estudio la que de acuerdo a la información obtenida era la más representativa. El empleo de la entrevista semiestructurada se debió a que permite mayor flexibilidad que la estructurada, además de que el entrevistador emplea una estrategia mixta (Díaz Bravo, Torruco García, Martínez Hernández, $\&$ Varela Ruiz, 2013). Se elaboró un guion de entrevista estructurada y semiestructurada usando como indicadores los objetivos de la economía social, además de las características de las empresas sociales presentadas en la tabla 1, debido a que permiten diferenciar a las empresas sociales del resto. Las personas entrevistadas fueron productores que se encontraban en una feria de economía solidaria y cooperativismo en la ciudad de Morelia, organizada en conjunto por la secretaria de desarrollo rural del estado de Michoacán y la organización promotora de la empresa social que fue objeto de estudio. Durante la investigación se realizaron 6 entrevistas estructuradas, 8 entrevistas semiestructuradas y 4 entrevistas no estructuradas. Previamente se 
LA EMPRESA SOCIAL: ACTOR CLAVE EN LA ECONOMÍA SOCIAL Y SOLIDARIA.

entrevistó a informantes clave durante otro encuentro de pequeños productores locales, donde uno de ellos refirió:

“hay 3 grupos de productores que producen orgánico, pero hay uno que producen orgánico y hacen economía social y comercio justo; hasta pintaron el pueblo y la plaza con lo que ganaron cuando se juntaron ellos, nada más que tienen sus propias ferias" E1.

En ese sentido, durante las entrevistas se pudo identificar 2 características principales que de acuerdo con la tabla 1 tienen las empresas sociales, las cuales son; el cuidado del medio ambiente y el desarrollo local de su comunidad. Otro entrevistado refirió:

“Ese grupo de productores tienen ya varios años (...) creo que la mayoría son mujeres, pero hacen muebles, mole y creo que también licores de frutas, hay otros, pero esos son los que más productores tienen y dicen que hacen economía social" E2.

Para esta investigación se eligió a una asociación de promotores de la empresa social; la cual es un organismo civil y altruista sin fines de lucro, que tiene como misión implementar la alternativa de la economía solidaria en las organizaciones productivas que favorezcan a las personas y a comunidades vulnerables, con el propósito de alcanzar equidad y justicia, y una adecuada distribución de la riqueza a través de la cadena productiva de cada bien y servicio. La organización otorga a sus integrantes asesoría y capacitación del trabajo comunitario en equipo y capitalización de remesas para proyectos productivos. Su visión es "unir al que produce con el que consume, evitando a los intermediarios, que funcionan como coyotes y obtienen las ganancias". Esta organización, logró identificar a migrantes que tenían deseos de hacer negocios entre México y los Estados Unidos de Norte América, mismos que tenían la capacidad de distribuir los productos del campo y las artesanías mexicanas en el país vecino, solo necesitaban organización, capacitación y solidaridad. Alrededor de 300 comunidades con más de 15,000 pequeños productores, organizados en 92 municipios de Michoacán, fueron capacitados y ayudados en ese entonces. Su fundador asegura que para que los proyectos se realicen y sea posible trabajar con las remesas, es necesario hacerlo bajo el modelo de la Economía Solidaria y Comercio Justo, erradicando a los intermediarios para evitar que mermen las ganancias y estas sean realmente repartidas entre los productores y los consumidores, trabajando de forma colectiva (López, 2009).

Actualmente de forma anual se realizan ferias de Comercio Justo y Economía Solidaria, en las que participan alrededor de 70 productores del interior del estado y del país, e incluso de estados TORRES-GONZÁLEZ K. E.

SEPTIEMBRE-DICIEMBRE 2020. Año 26, Número 76. Págs. 304-318 
LA EMPRESA SOCIAL: ACTOR CLAVE EN LA ECONOMÍA SOCIAL Y SOLIDARIA.

como Chiapas, estado de México, Distrito Federal, Jalisco, Guanajuato y Querétaro. Se realizaron entrevistas a profundidad, estructuradas y semiestructuradas las cuales contenían preguntas con indicadores basados en los principios, valores y objetivos de la economía social con el objetivo de conocer cómo operan estas empresas sociales.

\section{RESULTADOS}

Las entrevistas tenían por objetivo conocer si la forma en que operan las empresas pertenecientes a la organización que promueve la economía social y solidaria y el comercio justo fomentaba los objetivos, principios y valores de la economía social y solidaria y cómo actuaban en la práctica. Para lograr lo anterior, se elaboraron preguntas con 15 indicadores clave que corresponden a las características de la empresa social y a los valores que promueve la economía social y solidaria. Las 8 entrevistas estructuradas se realizaron durante una de las ferias que realiza la asociación en conjunto con el gobierno del estado de Michoacán. Las entrevistas contenían, entre otras, preguntas sobre cuidado del medio ambiente a lo que la mayoría de los entrevistados respondió positivamente incluso uno de ellos aseguró:

"Yo cuido la naturaleza porque vivo de ella, no está bien que queremos tener todo tipo de fruta y verduras todo el año, eso no es normal, la tierra también necesita descansar, ¿sabía eso?, el jitomate (...) no deberíamos tener jitomate todo el año, luego por eso todo lo comen lleno de químicos (...) yo tengo jitomate, pero también tengo otras verduras porque no me gusta usar químicos yo también como la verdura que vendo" E4.

De acuerdo con Vega (2014) y Collin (2014) en cuanto al cuidado del medio ambiente y compromiso con el entorno, se encontró que el productor busca una forma diferente de producir, por lo cual aseguraron no emplear agroquímicos y producir orgánicamente, esta consiente del cuidado del medio ambiente y también del tipo de relación entre productor y consumidor en la que ambos individuos se ven beneficiados, pues buscan ofrecer productos de calidad, aunque esto resulte en no poder ofertar a gran escala ni todo el año. De igual forma, otros productores refirieron cuidar el medio ambiente, plantando árboles, sembrando sin agroquímicos o bien cuidando el agua usándola moderadamente mediante sus propias técnicas de riego, además de no usar aguas negras para ello. Sin embargo, también se encontró que no todos los productores están conscientes de lo anterior, pues hubo quien refirió: "Si cuidamos el medio ambiente no tiramos basura y cuidamos el agua, pero aquí lo que interesa es que no haya intermediarios porque esos son los que se aprovechan de todos" E5. 
LA EMPRESA SOCIAL: ACTOR CLAVE EN LA ECONOMÍA SOCIAL Y SOLIDARIA.

No obstante, este productor no mencionó de forma concreta que acciones realiza para cuidar el medio ambiente en sus procesos de producción. Otros productores no respondieron específicamente con cuales acciones cuidan el medio ambiente. Por otra parte, las empresas sociales que promueven la economía social y solidaria tienen ciertas características como; procurar el desarrollo local, favorecer la creación de servicios sociales personales y la integración laboral de las personas. La mayoría de los productores entrevistados mencionaron que reciben "envíos de dinero" (dólares), ese dinero no solo lo emplean para cubrir sus necesidades básicas de alimento, salud y vivienda, también fueron alentados por la asociación y otros compañeros pertenecientes a la misma para capitalizar y producir sus propias pequeñas empresas. Esta asociación de promotores de la economía social, años atrás llamó la atención de diversos investigadores y ha sido objeto de diversos estudios debido a que en sus inicios fue fundada por un sacerdote que vio la necesidad del pueblo de mejorar su calidad de vida. Se buscó la forma de emplear las remesas que recibía el pequeño pueblo en el estado de Michoacán, organizados y dirigidos por el sacerdote, este grupo de productores logró crear empresas que incluso exportaban sus productos a los Estados Unidos de Norte América y con las ganancias obtenidas mejoraron su calidad de vida y la imagen de su pueblo, entre otros aspectos que abonaron a los procesos de desarrollo local. Con el fin de indagar si habría más casos similares al de este municipio, se cuestionó sobre este aspecto a los productores debido a que no solo pertenecen al estado de Michoacán sino también participan estados que se han ido uniendo a lo largo del tiempo como Jalisco, Colima, Guerrero, Oaxaca e incluso Chiapas. No obstante, los productores entrevistados solo refirieron que sus productos contribuyen al cuidado de la salud, medio ambiente y economía local puesto que están vendiendo sin intermediarios, por lo tanto, no se encontró que está experiencia se haya replicada en otras localidades de Michoacán o bien de los estados de los que provienen los productores. Por otra parte, durante las entrevistas varios productores mencionaron que emplean formas distintas al dinero de intercambio por sus productos:

"Nosotros no queremos depender del dinero para todo, porque no siempre tenemos, pero si yo tengo mangos y mi compañero tiene queso o jabón, ¿para qué queremos dinero?, podemos hacer "trueque", el trueque es un acuerdo, eso se usaba desde antes y así le hacemos en veces (...) todavía, es bueno" E6.

En ese sentido, de acuerdo con Collin y Torres (2007), The European Commission (2011), Vega (2014) y Gonzáles (2011), dentro de la economía social y solidaria existen formas alternativas de 
LA EMPRESA SOCIAL: ACTOR CLAVE EN LA ECONOMÍA SOCIAL Y SOLIDARIA.

circulación mismas que pueden ser compra y consumo de insumos en moneda alternativa, o bien el trueque, en ese sentido se encontró que entre los productores pertenecientes a la asociación es común esta práctica, sin que refirieran problemas o molestia por implementarla. Otro de los temas que se abordó durante las entrevistas fue la cooperación, a lo que los productores refirieron que se apoyan mutuamente de diversas formas debido a que es uno de los motivos por los cuales crearon la asociación, uno de los entrevistados refirió:

"Pues la cooperación está desde que puede ver que nos juntamos hacemos reuniones y pues aquí estamos (...) en que invitamos a productores de otros estados, hay compañeros que venden lo mismo (...) pero eso no importa, para todos hay (...) a lo mejor a uno no le gusta o no quiere lo que yo vendo pues que pruebe lo que tiene mi compañero, el chiste es apoyarnos o si a mí ya se me acabo el producto pues le puedo decir que vaya con mi compañero que todavía tiene y pues la gente sabe que aquí todo es de calidad" E7.

Finalmente, en cuanto a la integración laboral e igualdad, los productores mencionaron que ven en este tipo de empresas el trabajo adecuado para sus necesidades como refirió un productor:

"Para mí que soy mujer este tipo de trabajo es bueno porque pues yo tengo hijos y a veces no hallo con quien dejarlos (...) mi marido está en "el otro lado" y luego pues son 4 y mi hijo el mayor pues también va a la escuela y no puede cuidarlos todo el día, ya mejor aquí vendo mis productos y me los traigo, sirve que se enseñan a trabajar" E8.

Durante las entrevistas y observación en campo se encontró que la mayoría de los productores que participan en esta organización pertenecen al género femenino. También se encontró a productores con alguna situación de discapacidad, al cuestionarles sobre el tema, refirieron que están conscientes de que todas las personas merecen ser tratadas por igual y aseguraron estar dispuestos a emplear personas con alguna discapacidad, si esta no le impide cumplir con su trabajo, debido a que hay actividades en las cuales casi cualquier persona puede colaborar; de igual forma, aseguraron no tener ningún problema en emplear a personas mayores o diferenciar entre hombre y mujer. 


\section{CONCLUSIONES}

Esta investigación, se llevó a acabo usando el método cualitativo estudio de casos y se empleó como herramienta la entrevista sin estructura, semiestructurada y estructurada; se realizaron un total de 18 entrevistas a productores de una asociación promotora de la economía social en la ciudad de Morelia, Michoacán. Las entrevistas contenían preguntas elaboradas con base en los principios y valores de la economía social y las características de las empresas sociales debido a que es la forma en la que estas empresas se diferencias del resto. El objetivo principal de esta investigación fue, conocer como la empresa social coadyuva con la economía social. En ese sentido se encontró evidencia a favor en los siguientes aspectos:

Con respecto al cuidado del medio ambiente: los productores pertenecientes a la asociación promotora de la empresa social, aseguraron que el cuidado del medio ambiente es uno de los temas centrales bajo los cuales realizan sus prácticas de producción, llegando incluso a asegurar que no ofertan todo tipo de vegetales y frutas durante todo el año, aunque esto les represente una fuerte desventaja con respecto a la forma de producir tradicional en la que el consumidor puede acceder a casi cualquier tipo de productos durante todo el año. También afirmaron no emplear aguas negras y tener sus propias técnicas de riego con el fin de aminorar el gasto de agua.

Con respecto formas alternativas de circulación: los productores entrevistados aseguraron que además del dinero tienen por costumbre aceptar el intercambio de productos por lo que es común entre ellos el "trueque" como forma alternativa de circulación.

Con respecto a la cooperación: los productores entrevistados refirieron que este es uno de los principales motivos por los cuales se unieron, debido a que aseguraron que unidos era más fácil conseguir contactos para ofertar sus productos en otros lados. Por ejemplo, en Estados Unidos si era el caso, o bien buscando el apoyo del gobierno local para crear sus ferias y reuniones donde los consumidores pueden conocer sus productos e incluso ayudar en otras cuestiones, aunque no refirieron cuales.

Con respecto a la integración laboral: Este aspecto fue uno de los más interesantes de la investigación debido a que en un inicio esta asociación fue creada por personas que con las remesas que enviaban los migrantes a su familia, en su mayoría mujeres, lideradas por su fundador decidieron capitalizar esos recursos para crear empresas que incluso exportaban sus 
productos hacia Estados Unidos con la ayuda de los migrantes que vivían allá. Sin embargo, aunque actualmente participan productores de otros estados de la república mexicana, la mayoría de los productores siguen perteneciendo al género femenino, durante las entrevistas los productores refirieron que en este tipo de empresas habían encontrado el trabajo ideal pues les permitía cumplir con sus funciones como madres o padres a diferencia de los empleos capitalistas con largas jornadas laborales. Encuentran en este tipo de economía una alternativa que incluso procura a la familia. Aunque, en cuanto a los procesos de desarrollo local no se encontró que la primera experiencia en la que los productores se unieron para mejorar las condiciones de vida en su localidad, como fue el caso del municipio de Atacheo en el estado de Michoacán que fue donde se fundó la asociación, se haya replicado a otros municipios o bien a otros estados de la república mexicana, lo cual podría resultar bastante interesante y benéfico para más comunidades. Por lo tanto, si bien es cierto que la integración laboral, la igualdad y el respeto al medio ambiente, entre otros principios y valores, son elementos que inciden en el desarrollo local como tal no se encontró una experiencia adicional. En ese sentido, el por qué no se ha replicado esa experiencia abre una línea de investigación para futuros estudios. Los productores aseguraron implementar la economía social y solidaria además del comercio justo en sus prácticas de comercio. No obstante, durante las entrevistas los productores se referían a la economía social y solidaria como a la eliminación de intermediarios, lo cual, si bien es cierto que es uno de los objetivos, no es el principal objetivo de este tipo de economía. Se puede concluir, que la economía social y solidaria y la empresa social, se encuentra en desarrollo en el estado de Michoacán y aunque cada vez se agregan más productores a la práctica, aún se precisa más trabajo al respecto sobre todo en reproducir casos de éxito como los ocurridos en la comunidad donde se generó la asociación más grande que promueve la economía social y solidaria. Por otra parte, es preciso establecer otra manera de ofertar los productos localmente, debido a que los consumidores en su mayoría tienen que esperar a que se reúnan en ferias y encuentros, ello genera dificultades para el consumidor que requiere de ciertos productos con determinada periodicidad y esto representa una clara desventaja frente a las empresas capitalistas. Finalmente, en cuanto al papel que juega la empresa social en este tipo de economía, se encuentra que esta funge como el medio primordial mediante el cual tanto productores como consumidores pueden establecer relaciones de reciprocidad e incluso en pro de sus comunidades y el medio ambiente. Por tanto, con base en los resultados se puede concluir que la economía social y solidaria precisa y encuentra en este tipo de empresas un vínculo entre productor y consumidor que permite promover la práctica y desarrollo de este tipo de economía. 


\section{REFERENCIAS BIBLIOGRÁFICAS}

Barrio, I., González, J., Padín, L., Peral, P., Sánchez, I. \& Tarín, E. (2016). El Estudio de Casos. Métodos de investigación educativa. Universidad Autónoma de Madrid. España: Madrid.

Collin, L. (2008). La economía social y solidaria. Revista Pasos, 135.

Collin, L. (2014). Economía solidaria: local y diversa. Tlaxcala México: El Colegio de Tlaxcala, Centro Argentino de Etnología Americana.

Collin, L. \& Torres, A. (2007). Normas para la operación de empresas sociales. Artículo inédito.

Coraggio, J. L. (2004). Economía del trabajo: una alternativa racional a la incertidumbre. Íconos, 29-38.

Coraggio, J. L. (2016). Economía social y solidaria en movimiento. Buenos Aires, Argentina: Ediciones UNGS. Universidad Nacional de General Sarmiento.

Díaz, L., Torruco, U., Martínez, M. \& Varela, M. ( 2013). La entrevista, recurso flexible y dinámico. Metodología de investigación en educación médica, 162-167. Universidad Nacional Autónoma de México, D.F.: México.

Díaz, M. \& Marcuello, C. (2014). Las empresas sociales en España:. Gizarte Ekonomiaren Euskal Aldizkaria- Revista Vasca de Economía Social, 143-164. Universidad de Zaragoza.

García, A. (2011). El comercio justo: ¿una alternativa de desarrollo local? Revista Polis, 105-140. Universidad Autónoma Metropolitana.

Girona, E. \& Sanchis, J. (2014). Las empresas sociales y el management social. Gizarte Ekonomiaren Euskal Aldizkaria-Revista Vasca de Economía Social, 189-216. Universidad de Valencia.

Gonzáles, M. A. (2011). Economía social para la vida.Desafíos a la educación. Saberes para la acción en educación de adultos, 3-9. Obtenido de https://www.crefal.org/decisio/images/pdf/decisio_29/decisio29_saber1.pdf

López, C. (15 de Octubre de 2009). El padre Marcos y la economía solidaria «Que el que consume defienda al que produce». Recuperado el 24 de Septiembre de 2016, de El Portal de economía solidaria: https://www.economiasolidaria.org/noticias/el-padre-marcos-y-laeconomia-solidaria-que-el-que-consume-defienda-al-que-produce

Monzón, J. L. (2006). Economía Social y conceptos afines: fronteras borrosas y ambigüedades conceptuales del Tercer Sector. CIRIEC-Eapaña-Revista de economía pública, social y cooperativa., 9-24. Recuperado el 15 de abril de 2019, de https://base.socioeco.org/docs/a7405602.pdf

Pérez de Mendiguren, J. C., Etxarri, E. \& Guridi, L. (2008). ¿De qué hablamos cuando hablamos de Economía Social y Solidaria? Concepto y nociones afines. XI Jornadas de Economía Crítica, 1-26. 
LA EMPRESA SOCIAL: ACTOR CLAVE EN LA ECONOMÍA SOCIAL Y SOLIDARIA.

Razeto, L. (Diciembre de 2014). ¿Qué es la economía solidaria?, por Luis Razeto. Recuperado el 12 de 04 de 2019, de Portal de economía solidaria: https://www.economiasolidaria.org/reas-red-de-redes-de-economia-alternativa-ysolidaria/biblioteca/que-es-la-economia-solidaria-por

Tapia, R. (03 de Septiembre de 2012). Economía solidaria: una alternativa de desarrollo local integral en América Latina. Recuperado el 23 de Febrero de 2019, de Portal de economía solidaria: https://www.economiasolidaria.org/noticias/economia-solidaria-una-alternativade-desarrollo-local-integral-en-america-latina

The European Commission. (2011). What are social enterprises? Recuperado el 12 de Junio de 2019, de The Social Business Initiative of the European Commission: https://ec.europa.eu/growth/sectors/social-economy/enterprises_en

Vega , L. (2014). Economía social y. Recuperado el 06 de Marzo de 2019, de ConectaDEL: http://www.conectadel.org/wp-content/uploads/downloads/2015/08/9-Rev-Paco-Eco-Soly-DL-_20_02_15.pdf

Yin, R. (1992). The case study method as a tool for doing evaluation. Current sociology, 121-137. 